\title{
Treatment of experimental autoimmune myasthenia gravis rats with FTY720 and its effect on Th1/Th2 cells
}

\author{
JIANKANG HUANG, TING ZHANG, HONGMEI WANG and YUWU ZHAO \\ Department of Neurology, Shanghai Jiaotong University Affiliated Sixth People's Hospital, Shanghai 200233, P.R. China
}

Received January 4, 2017; Accepted November 15, 2017

DOI: $10.3892 / \mathrm{mmr} .2018 .8768$

\begin{abstract}
Myasthenia gravis (MG) is an autoimmune neurological disease that is characterized by the expression of anti-acetylcholine receptor (AChR) antibodies. The immune response at AChRs of neuromuscular junction is disrupted in patients with MG, which manifests as skeletal muscle fatigue and is aggravated following periods of activity and alleviated following rest. Although a novel immune suppressant FTY720 drug, which exhibits strong immune suppression efficacy and minor adverse effects, is available, its role and mechanism in MG have not been elucidated. The aim of this study was to investigate the role of FTY720 in MG. A total of 60 healthy female Lewis rats were randomly assigned into 4 groups: Control group, Model group of experimental autoimmune myasthenia gravis (EAMG), $0.5 \mathrm{mg} / \mathrm{kg}$ FTY720-treatment EAMG group and $1.0 \mathrm{mg} / \mathrm{kg}$ FTY720-treatment EAMG group. Body weight and symptoms were examined; Lennon score was used to evaluate improvement of clinical symptoms. Reverse transcription-quantitative polymerase chain reaction and ELISA were used to test the mRNA and protein expression levels, respectively, of the helper $\mathrm{T}$ (Th) 1 and Th2 cell cytokines, including interleukin (IL)-2, interferon (IFN)- $\gamma$, IL- 4 and IL- 6 in thymus tissue and serum. FTY720 treatment improved rat MG symptoms, increased body weight and decreased Lennon score. FTY720 treatments also reduced tissue and serum levels of IL-2, IFN- $\gamma$ and IL-6, but not IL-4 expression levels. FTY720 suppressed the inflammatory response and improved EAMG symptoms by inhibiting the secretion of inflammatory factors.
\end{abstract}

\section{Introduction}

Myasthenia gravis (MG) is a common autoimmune neurological disorder, with an incidence rate between 7.7

Correspondence to: Dr Yuwu Zhao, Department of Neurology, Shanghai Jiaotong University Affiliated Sixth People's Hospital, 600 Yishan Road, Shanghai 200233, P.R. China

E-mail: yuwuzhaoasd@163.com

Key words: FTY720, myasthenia gravis, inflammatory factor, $\mathrm{T}$ helper 1, T helper 2 and 15 per 100,000 across all age groups, but with a female bias $(1,2)$. MG is mainly induced by production of an acetylcholine receptor (AChR) antibody, which may cause abnormal immune responses of $\mathrm{AChR}$ at neuromuscular junctions, thus depriving its normal neural transmission function (3). Clinical manifestation of MG is general skeletal muscle fatigue and blepharoptosis, both of which are aggravated following periods of activity and alleviated with rest, thus exhibiting relief in the morning and progression of symptoms in night (4). MG progression may affect neural tissues other than neuromuscular junction or may affect non-neural tissues, which may lead to epilepsy, pyramidal tract sign and memory deficit $(5,6)$. The pathogenic mechanism of MG is complex and involves genetic, environmental, physical and chemical factors (7). As an autoimmune disease, MG also involves abnormality in humoral immunity and cellular immunity response $(8,9)$.

Thelper (Th) cells are subdivided into two sub-populations, Th1 and Th2, based on the differential secretion of cytokines (10). A recent study demonstrated that Th1 and Th2 cells maintain the Th1/Th2 balance by regulating cytokine secretion, thus serving a crucial role in maintaining normal immune functions (11). Th1 cells mainly secrete interleukin (IL)-2 and interferon (IFN)- $\gamma$, whereas Th2 cells mainly produce IL-4 and IL-6 cytokines (12). Through self-regulation and cross-regulation of secreted cytokines, Th1 and Th2 maintain a homeostasis and server crucial roles in regulating both cellular and humoral immunity (13). Another recent study demonstrated the role of Th1/Th2 secreted factors in the occurrence and progression of MG (14). FTY720, also known as fingolimod, is a new generation immune suppressant that is extracted from the Traditional Chinese Medicine Cordyceps sinensis, with the major component ISP-I (also known as myriocin) having immune suppressing roles that induce lymphocyte apoptosis, accelerate matured lymphocyte nesting and inhibit translocation of $\mathrm{T}$ cells from the thymus to the peripheral blood circulation, and thus serving a role in transplantation immunity with strong effects and minor side effects (15). A recent study confirmed the role of FTY720 in regulating autoimmune diseases such as encephalomyelitis (16); however, the role of FTY720 in MG remained unknown. The aim of the present study was to investigate the role of FTY720 in MG and its underlying therapeutic mechanism, as well as providing a basis for the discovery of novel treatment approaches for MG. 


\section{Materials and methods}

Experimental animal selection. A total of 60 healthy female specific-pathogen-free (SPF)-grade Lewis rats (age, 2-3 months; weight, $130 \pm 20 \mathrm{~g}$ ) were purchased from the Laboratory Animal Center of Shanghai Jiaotong University (Shanghai, China) and were maintained in an SPF-grade facility at a fixed temperature of $21 \pm 1^{\circ} \mathrm{C}$, relative humidity between 50 and $70 \%$ and a $12 \mathrm{~h}$ light/dark cycle with free access to food and water. This study was approved by the Laboratory Animal Management and Ethics Committee and followed animal welfare codes in Shanghai Jiaotong University Affiliated Sixth People's Hospital.

Materials and equipment. Pentobarbital sodium and lidocaine were purchased from Shanghai Zhaohui Pharmaceutical Co., Ltd. (Shanghai, China). FTY720 was purchased from Jiangxi Haoran Bio-Pharma Co., Ltd. (Jiangxi, China). R-AChR subunit $\alpha$ region 97-116 was purchased from Xi'an Lianmei Biotechnology Co.,Ltd.(Xi'an, China). Complete Freund's adjuvant (CFA) was purchased from Sigma-Aldrich (Merck KGaA, Darmstadt, Germany). ELISA kits for IL-2 (cat. no. R2000; Rat IL-2 Quantikine ELISA Kit), IFN- $\gamma$ (cat. no. RIF00; Rat IFN-gamma Quantikine ELISA Kit), IL-4 (cat. no. R4000; Rat IL-4 Quantikine ELISA Kit) and IL-6 (cat. no. R6000B; Rat IL-6 Quantikine ELISA Kit) were purchased from R\&D Systems, Inc. (Minneapolis, MN, USA). TRIzol reagent, Total RNA Extraction kit, reverse transcription-quantitative polymerase chain reaction (RT-qPCR) primers, and High-Capacity cDNA Reverse Transcription kit were purchased from Invitrogen (Thermo Fisher Scientific, Inc., Waltham, MA, USA). A LabSystem 1.3.1 microplate reader was purchased from Bio-Rad Laboratories, Inc. (Hercules, CA, USA). The ABI 7700 Fast Real-Time PCR cycler was purchased from Applied Biosystems (Thermo Fisher Scientific, Inc.). An ultrapure workstation was purchased from Sutai High-Tech Materials Co., Ltd. (Shanghai, China).

Animal grouping and treatment. Healthy female SPF-grade Lewis rats were randomly assigned into 4 groups ( $\mathrm{n}=15 /$ group): i) Control group, which received no treatment; ii) experimental autoimmune myasthenia gravis (EAMG) model group, which received triplicate subcutaneous injections of the immunogen R-AchR- $\alpha 97-116$ (200 $\mu 1$ of CFA per injection at each body site) at 3 different body sites (tail, back and foot) on days 0,30 and 60 ; iii) $0.5 \mathrm{mg} / \mathrm{kg}$ FTY720 group, which received FTY720 $(0.5 \mathrm{mg} / \mathrm{kg})$ by gavage 15 days post-EAMG immunization; and iv) $1.0 \mathrm{mg} / \mathrm{kg}$ FTY720 group, which received FTY720 $(1.0 \mathrm{mg} / \mathrm{kg}) 15$ days post-EAMG immunization. The EAMG model was prepared via injection in the rats with immunogen R-AchR- $\alpha$ 97-116 on days 0,30 and 60 using the same method.

Observation of symptoms and Lennon score. Lennon scoring was used to evaluate EAMG model rats in a double-blinded manner (17). Major symptoms of EAMG include muscle weakness, shakiness, body curvature, fatigue and hoarse vocalization. The clinical evaluation was performed based on the EAMG clinical grading scales in animal models (rat), which were developed by Lennon et al (18). A score of 0 was defined as normal, in which rats exhibited muscle strength without
Table I. Primer sequences for reverse transcription-quantitative polymerase chain reaction.

\begin{tabular}{ll}
\hline Gene & \multicolumn{1}{c}{ Primer sequence $\left(5^{\prime} \rightarrow 3^{\prime}\right)$} \\
\hline GAPDH & F: AGTGCCAGCCTCGTCTCATAG \\
& R: CGTTGAACTTGCCGTGGGTAG \\
IL-2 & F: CCAGGATAGCGATACGAACTT \\
& R: GGCATCTCTGCTTCAACCG \\
IFN- $\gamma$ & F: CGGCTAAGAAATAGCGATCTC \\
& R: GGCTCAATCGCTCTCGACT \\
IL- 4 & F: GGATCTAGCCGGAACAATACT \\
& R: CGTCTGACATCTGGCAGGAT \\
IL-6 & F: GAAGATCTCAATAGCACGT \\
& R: AATCTCTCACGTCATCCTTC
\end{tabular}

IFN, interferon; IL, interleukin; F, forward; R, reverse.

abnormality. A score of 1 was assigned to rats exhibiting mild abnormality of muscle strength, minor decrease of activity, weakness of grasping and fatigue. A score of 2 was assigned to rats that exhibited significant abnormality in motility, decreased activity or body weight, head/tail falling, unstable walking, body curvature under resting conditions, curved forelimbs and shaking. A score of 3 was assigned to rats that exhibited whole body weakness, no activity, body shakiness and dying status, which were consistent with previous studies $(18,19)$; a score of 4 was defined as death. Rats exhibiting boundary phenotypes were assigned an additional 0.5 point. All rats were weighted prior to tissue collection. The humane cut-off points were established according to a previous study (19).

Sample collection. A total of 20 days post-EAMG immunization, a blood sample $(5 \mathrm{ml})$ was collected from the tail vein from rats in each of the 4 groups. Blood samples were centrifuged at $3,000 \mathrm{x} \mathrm{g}$ for $15 \mathrm{~min}$, and serum was collected and stored at $-80^{\circ} \mathrm{C}$ for further assays. Subsequently, rats were sacrificed and thymus tissues were collected and stored at $-80^{\circ} \mathrm{C}$.

ELISA for serum levels of IL-2, IFN- $\gamma, I L-4$ and IL-6. Serum samples were examined for the expression levels of IL-2, IFN- $\gamma$, IL-4 and IL-6 using ELISA, following the manufacturer's protocol. Using a blank control well as the reference, absorbance values at $450 \mathrm{~nm}$ were measured by a microplate reader. A linear regression model was plotted based on the concentration of standard samples and respective optical density (OD) values. Sample concentration was further deduced based on OD values and regression function.

RT-qPCR for expression of Th1 and Th2 cytokines. Total RNA was extracted from thymus tissue samples $(100 \mathrm{mg})$ of rats using TRIzol reagent. Total RNA purity and quantification was examined by UV spectrometry. cDNA was synthesized by reverse transcription using High-Capacity cDNA Reverse Transcription Kit (Thermo Fisher Scientific, Inc.) according to the manufacturer's instructions via incubation for $5 \mathrm{~min}$ at $95^{\circ} \mathrm{C}$ followed by $5 \mathrm{~min}$ at $4^{\circ} \mathrm{C}$. Primer Premier 6.0 (Premier Biosoft International, Palo Alto, CA, USA) was used to design 


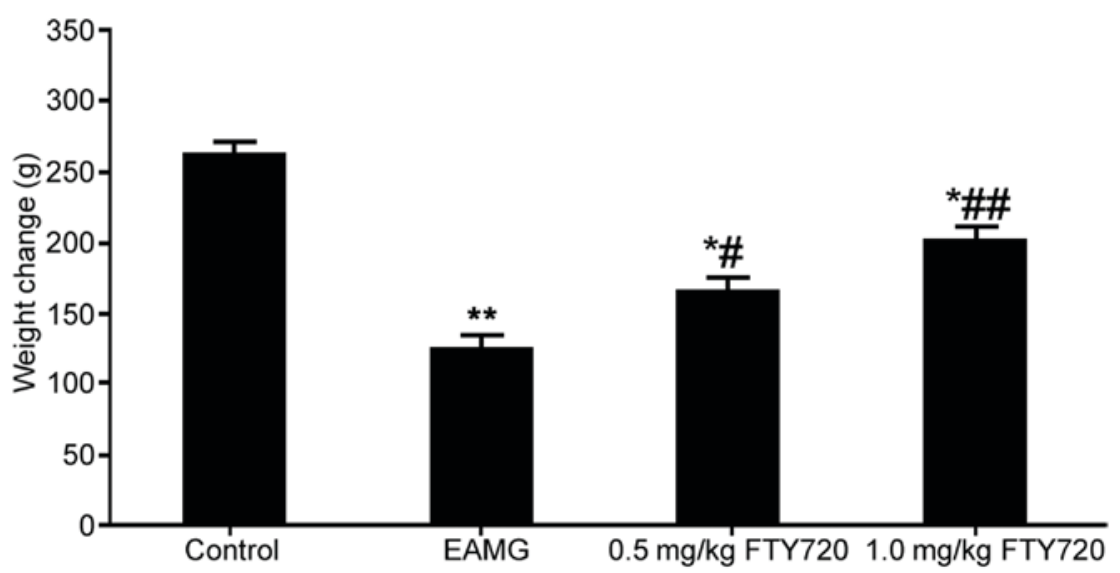

Figure 1. Body weight comparison of EAMG rats. Data are presented as the mean \pm standard deviation; $n=15 /$ group; ${ }^{*} \mathrm{P}<0.05$, ${ }^{* *} \mathrm{P}<0.01$ vs. Control; ${ }^{*} \mathrm{P}<0.05$, ${ }^{\#} \mathrm{P}<0.01$ vs. EAMG. EAMG, experimental autoimmune myasthenia gravis.

gene-specific PCR primers (Table I). qPCR was used to determine target gene mRNA expression levels using the following thermocycling conditions: 1 cycle at $92^{\circ} \mathrm{C}$ for $30 \mathrm{sec}$, followed by 35 cycles of $92^{\circ} \mathrm{C}$ for $30 \mathrm{sec}, 58^{\circ} \mathrm{C}$ for $45 \mathrm{sec}$ and $72^{\circ} \mathrm{C}$ for $35 \mathrm{sec}$, and final extension step at $72^{\circ} \mathrm{C}$ for $1 \mathrm{~min}$. Cq values were calculated based on internal reference gene GAPDH for plotting standard curve, and gene expression analysis was performed by the $2^{-\Delta \Delta \mathrm{Cq}}$ method (20).

Statistical analysis. SPSS 19.0 statistical software (IBM Corp., Armonk, NY, USA) was used for statistical analysis. Data are presented as the mean \pm standard deviation. Comparison of means among multiple groups was performed by one-way analysis of variance with Tukey's post hoc test. $\mathrm{P}<0.05$ was considered to indicate a statistically significant difference.

\section{Results}

Clinical symptoms, body weight and Lennon score. Healthy female SPF-grade Lewis rats were randomly assigned into four groups, including a Control group, an EAMG model group, which received injection of the R-AchR- $\alpha 97-116$ immune antigen, and two groups of EAMG rats that received either a low dose $(0.5 \mathrm{mg} / \mathrm{kg})$ or a high dose $(1.0 \mathrm{mg} / \mathrm{kg})$ of FTY720 15 days post-immunization. Following the second immunization, rats exhibited signs of muscle weakness, whereas typical MG symptoms occurred following the third round of immunization injections. EAMG model rats exhibited severe mental retardation, loss of glossy fur or even detachment, hypo-activity, limb weakness, weakened grasping, decreased appetite, body shaking and aggravated lower muscle strength following activity. FTY720 treatment slowed the onset of disease and alleviated symptoms, with more potent effects observed in the high-dosage group. EAMG model rats exhibited significantly lower body weight compared with the control group $(\mathrm{P}<0.01$; Fig. 1). EAMG rats treated with either a low or high dose of FTY720 had significantly higher body weights compared with untreated EAMG rats $(\mathrm{P}<0.05$ and $\mathrm{P}<0.01$, respectively; Fig. 1). The maximum body weight loss was $60 \mathrm{~g}$ (23\% total body weight), which was contemplated in the documents submitted to the Ethics Committee of the Shanghai

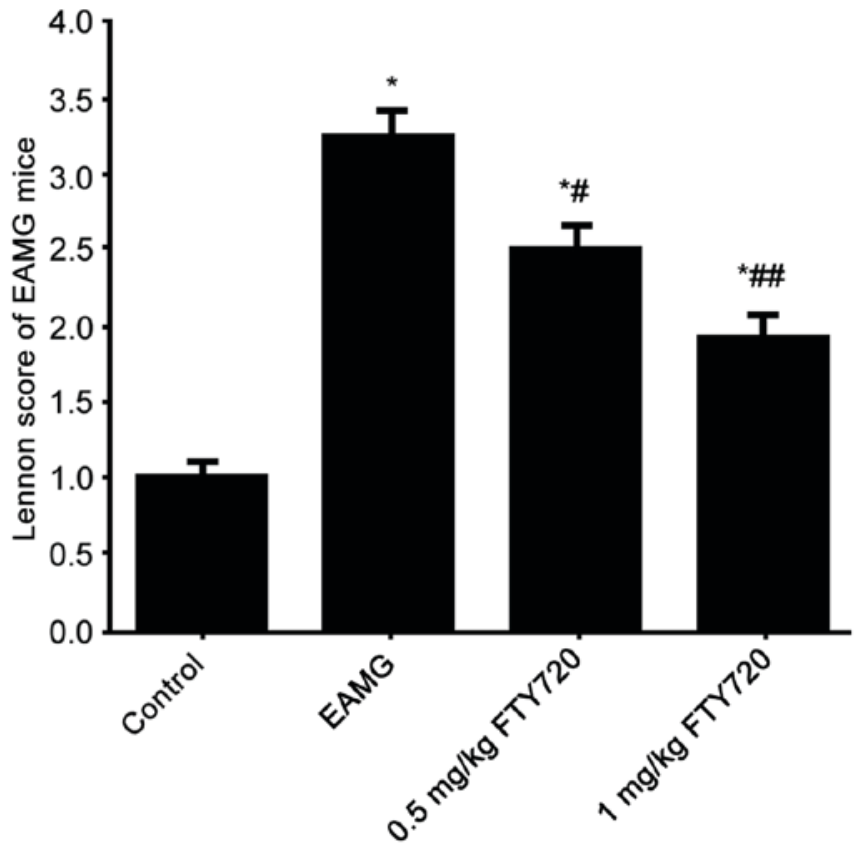

Figure 2. Effects of FTY720 treatment on Lennon score of EAMG model rats. ${ }^{*} \mathrm{P}<0.05$ vs. Control, ${ }^{\#} \mathrm{P}<0.05,{ }^{\# \#} \mathrm{P}<0.01$ vs. EAMG. EAMG, experimental autoimmune myasthenia gravis.

Jiaotong University Affiliated Sixth People's Hospital for approval.

Lennon score of all rats (score was defined as 1 in all control rats, data not shown) was evaluated. Rats in the EAMG group demonstrated significantly elevated Lennon scores compared with control rats (Fig. 2). FTY7210 treatment significantly suppressed Lennon scores in a dose-dependent manner compared with both the control group and the EAMG group (Fig. 2). These results indicated that FTY720 treatment may improve the clinical symptoms EAMG.

Effects of FTY720 treatment on mRNA expression levels of Thl cytokines IL-2 and IFN- $\gamma$ in thymus. RT-qPCR was used to examine mRNA expression levels of Th1 cytokines IL-2 and IFN- $\gamma$ in rat thymus tissues. In EAMG model rats, 


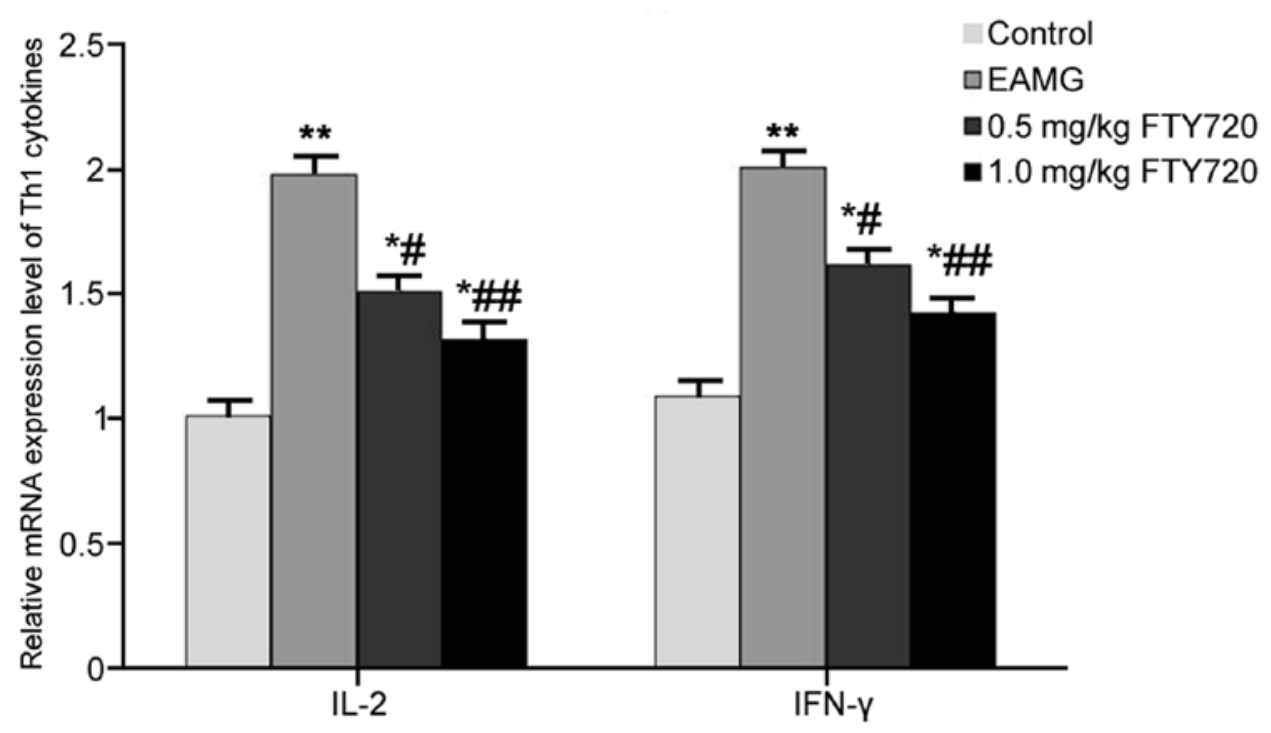

Figure 3. Effects of FTY720 treatment on Th1 cytokine mRNA expression levels in thymus tissues. mRNA expression levels of Th1 cytokines IL-2 and IFN- $\gamma$ were measured by reverse transcription-quantitative polymerase chain reaction. ${ }^{*} \mathrm{P}<0.05,{ }^{* * *} \mathrm{P}<0.01 \mathrm{vs}$. Control; ${ }^{\#} \mathrm{P}<0.05$, ${ }^{\# \#} \mathrm{P}<0.01 \mathrm{vs}$. EAMG. EAMG, experimental autoimmune myasthenia gravis; IFN, interferon; IL, interleukin; Th1, Thelper 1 cell.

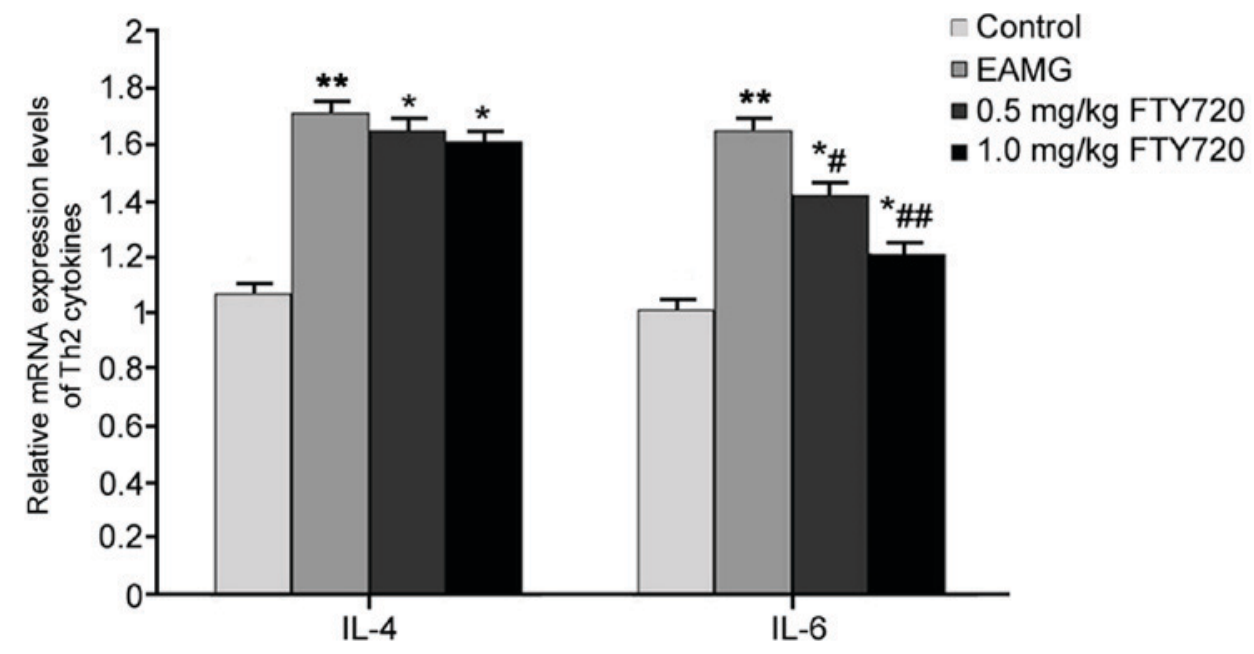

Figure 4. Effects of FTY720 treatment on Th2 cytokine mRNA expression levels in thymus tissues. mRNA expression levels of Th2 cytokines IL-4 and IL-6 were measured by reverse transcription-quantitative polymerase chain reaction. ${ }^{*} \mathrm{P}<0.05,{ }^{* *} \mathrm{P}<0.01$ vs. Control; ${ }^{\#} \mathrm{P}<0.05,{ }^{\# \#} \mathrm{P}<0.01$ vs. EAMG. EAMG, experimental autoimmune myasthenia gravis; IL, interleukin; Th2, T helper 2 cell.

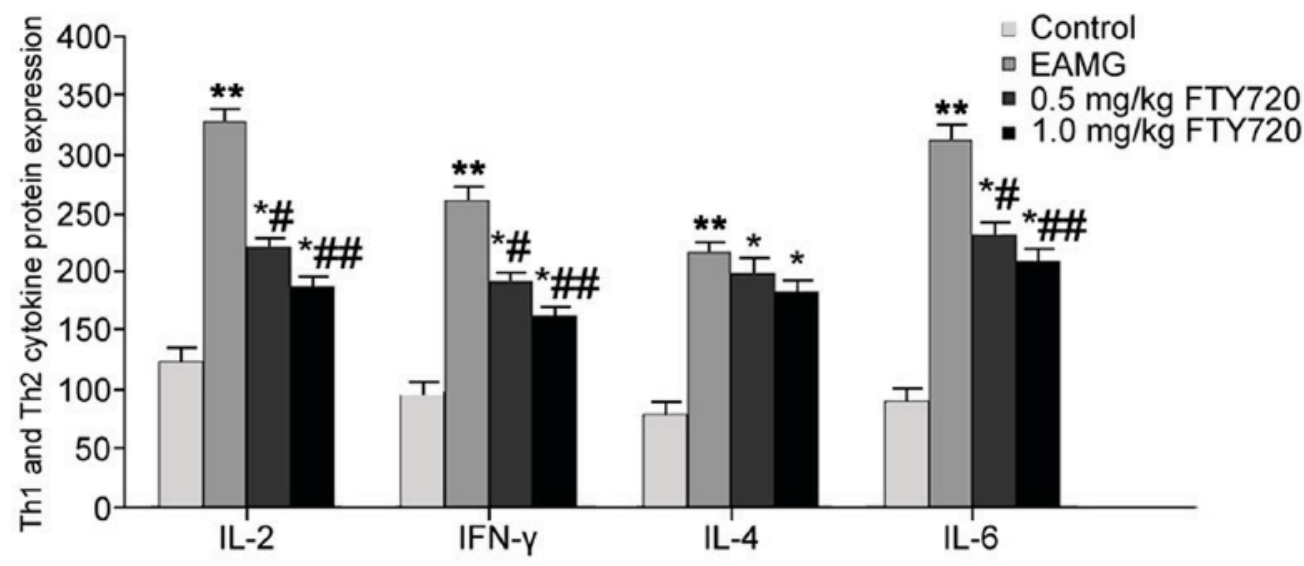

Figure 5. Effects of FTY720 treatment on expression levels of Th1 and Th2 cytokines in rat serum. Protein expression levels of Th1 cytokines IL-2 and IFN- $\gamma$ and Th2 cytokines IL- 4 and IL-6 were measured by ELISA. ${ }^{*} \mathrm{P}<0.05,{ }^{* *} \mathrm{P}<0.01$ vs. Control; ${ }^{\#} \mathrm{P}<0.05,{ }^{\# \#} \mathrm{P}<0.01$ vs. EAMG. EAMG, experimental autoimmune myasthenia gravis; IFN, interferon; IL, interleukin; Th, T helper cell. 
the mRNA expression levels of IL- 2 and IFN- $\gamma$ were significantly higher compared with the respective expression levels in Control rats $(\mathrm{P}<0.01$; Fig. 3). Low-dose FTY720 treatment significantly decreased IL-2 and IFN- $\gamma$ mRNA expression in the thymus tissues compared with expression in the EAMG model group $(\mathrm{P}<0.05$; Fig. 3$)$. Rats in the high-dosage FTY720 treatment group exhibited more potent inhibitory effects on IL- 2 and IFN- $\gamma$ mRNA expression levels $(\mathrm{P}<0.01$ vs. EAMG; Fig. 3).

Effects of FTY720 treatment on mRNA expression levels of Th2 cytokines IL-4 and IL-6 in thymus. RT-qPCR was used to examine the mRNA expression levels of Th2 cytokines IL-4 and IL-6 in rat thymus tissues. In EAMG model rats, the mRNA expression levels of IL- 4 and IL- 6 were significantly elevated compared with the respective expression levels in Control rats $(\mathrm{P}<0.01$; Fig. 4). Rats in the low-dose FTY720 treatment group exhibited a significantly decreased expression of IL-6 mRNA compared with untreated EAMG model rats $(\mathrm{P}<0.05$; Fig. 4), and high-dose FTY720 treatment had a more potent inhibitory effect on IL- 6 mRNA $(\mathrm{P}<0.01$ vs. EAMG). However, neither of the FTY720 treatments significantly affected IL-4 mRNA expression ( $\mathrm{P}>0.05$ vs. EAMG; Fig. 4).

Effects of FTY720 treatment on protein expression levels ofTh1 and Th2 cytokines in serum. ELISA analysis was used to determine the effects of FTY720 treatments on Th1 and Th2 cytokines in EAMG rat serum. In EAMG model rats, serum levels of IL-2, IFN- $\gamma$, IL- 4 and IL- 6 were significantly elevated compared with protein expression levels in Control group rats $(\mathrm{P}<0.01)$. IL-2, IFN- $\gamma$ and IL-6 expression levels were significantly reduced following low-dose FTY720 treatment $(\mathrm{P}<0.05$ vs. EAMG), with more potent inhibitory effects observed in the high-dosage FTY720 treatment group $(\mathrm{P}<0.01$ vs. EAMG). No significant differences were identified for either high- or low-dose FTY720 treatment on IL-4 expression levels, compared with EAMG model rats (Fig. 5).

\section{Discussion}

A previous study has demonstrated the close correlation between MG pathogenesis and humoral and cellular immune functions, particularly for cellular immunity (21). The Th1-induced immune response mainly serves a role in pathogenic immune responses by increasing IL-2 or IFN- $\gamma$ secretion, thus activating inflammatory response, whereas the Th2 subpopulation of $\mathrm{T}$ cells exerts protective functions through the secretion of cytokines that induce an immune response (22). During MG pathogenesis, both Th1 and Th2 cells are able to regulate MG progression through the synergistic effects of the secreted cytokines, with more potent effects being attributed to Th1 cytokines (23).

The present study used a R-AChR- $\alpha 97-116$ immunogen to generate EAMG model rats, in which the effects of treatment with the novel immune suppressant drug FTY720 were examine. The present results indicated that FTY720 treatment was able to reduce muscle weakness symptoms of EAMG rats, increase body weight and decrease Lennon score, which indicated that FTYP720 treatment may be used to examine the mechanisms of MG development, as well as a potential immune therapy. Previous results confirmed that Th1 cells secreted IFN- $\gamma$ to facilitate antigen-specific Th1 cell to generate AchR- $\alpha$ antibodies, the levels of which are increased in muscle and thymus epithelium cells and thus indicate a correlation between IFN- $\gamma$ and MG production (24). Th1 secreted IL-2 cytokines, which may further facilitate the activation of Th cells and natural killer cells, lead to cytokine production, as indicated by decreased IL-2 levels in MG patients (25). Th2-cell secreted self-activating factor IL-4 is an important factor for inducing the humoral immune response and is important for AchR- $\alpha$ antibody production, thus serving a crucial role in MG pathogenesis (26); however its functional mechanism remains unknown. Expression levels of the Th2-cell secreted IL-6 cytokine and its receptors have been reported to be upregulated in patients with $\mathrm{MG}$, which suggested that IL-6 receptor upregulation may activate $T$ cells and facilitate MG pathogenesis. In clinical assessments, increase in IL-6 activity or content may be used as a clinical marker for MG $(27,28)$. The present study analyzed the effects of FTY720 treatment on Th1 and Th2 cytokine production, and confirmed that FTY720 treatment was able to suppress IL-2, IFN- $\gamma$ and IL-6 expression levels in thymus tissues and sera, but did not affect IL-4 levels.

Although the present study obtained significant results, there were also a few limitations. First, the specific pharmaceutical mechanisms for the function of FTY720 have not been fully investigated, which maybe clarified in future studies. Second, the sample size of the experimental animals used was relatively small. Third, the protective function of FTY720 has not been investigated in other animal models, which may also be examined in the future studies.

In conclusion, FTY720 treatment suppressed the inflammatory response by inhibiting the secretion of inflammatory factors, thus improving the symptoms of EAMG. Results from the present study may provide additional evidence for EAMG pathogenesis mechanism and clinical treatment.

\section{Acknowledgements}

Not applicable.

\section{Funding}

This study was supported by The Medical Professional Cross Research Fund, Project of Shanghai Jiao Tong University (grant no. YG2015MS14).

\section{Availability of data and materials}

The datasets used and/or analyzed during the current study are available from the corresponding author on reasonable request.

\section{Authors' contributions}

JH substantially contributed to the design of the study and the performance of the experiments. YZ, TZ and HW contributed 
to acquisition, analysis and interpretation of the data. YZ provided final approval of the version of the manuscript to be published and agreed to be accountable for all aspects of the work in ensuring that questions related to the accuracy or integrity of any part of the work are appropriately investigated and resolved.

\section{Ethics approval and consent to participate}

This study has been reviewed and approved by the Ethical Committee of Shanghai Jiaotong University Affiliated Sixth People's Hospital (Shanghai, China).

\section{Consent for publication}

Not applicable.

\section{Competing interests}

The authors declare that they have no competing interests.

\section{References}

1. Lizarraga AA, Lizarraga KJ and Benatar M: Getting rid of weakness in the ICU: An updated approach to the acute management of myasthenia gravis and guillain-barré syndrome. Semin Neurol 36: 615-624, 2016.

2. Sonkar KK, Bhoi SK, Dubey D, Kalita J and Misra UK: Direct and indirect cost of myasthenia gravis: A prospective study from a tertiary care teaching hospital in India. J Clin Neurosci 38: 114-117, 2016.

3. Beck G, Yabumoto T, Baba K, Sasaki T, Higuchi O, Matsuo H and Mochizuki H: Double seronegative myasthenia gravis with anti-LRP4 antibodies presenting with dropped head and acute respiratory insufficiency. Intern Med 55: 3361-3363, 2016.

4. Xin Y, Cai H, Lu T, Zhang Y, Yang Y and Cui Y: miR-20b Inhibits T Cell Proliferation and Activation via NFAT signaling pathway in thymoma-associated myasthenia gravis. Biomed Res Int 2016: 9595718, 2016.

5. Lee HE, Kim YH, Kim SM and Shin HY: Clinical significance of repetitive compound muscle action potentials in patients with myasthenia gravis: A predictor for cholinergic side effects of acetylcholinesterase inhibitors. J Clin Neurol 12: 482-488, 2016.

6. Gung Y, Zhang H, Li S and Wang Y: Sternotomy versus video-assisted thoracoscopic surgery for thymectomy of myasthenia gravis patients: A meta-analysis. Asian J Endosc Surg 9: 285-294, 2016.

7. Meng QF, Zhang Z, Wang YJ, Chen W, Li FF, Yue LT, Zhang CJ, $\mathrm{Li} \mathrm{H}$, Zhang M, Wang CC, et al: Astilbin ameliorates experimental autoimmune myasthenia gravis by decreased Th17 cytokines and up-regulated T regulatory cells. J Neuroimmunol 298: 138-145, 2016.

8. Zhang H, Geng Y, Zheng Y and Wang Y: A case of anterior mediastinitis and bilateral multiple lung abscesses occurring after trans-subxiphoid video-assisted thoracoscopic extended thymectomy for thymoma with myasthenia gravis. J Thorac Dis 8: E970-E973, 2016.

9. Nikolic AV, Bojic SD, RakocevicStojanovic VM, Basta IZ and Lavrnic DV: Electrophysiological findings in patients with low density lipoprotein receptor related protein 4 positive myasthenia gravis. Eur J Neurol 23: 1635-1641, 2016.

10. Vargas-Rojas MI, Solleiro-Villavicencio H and Soto-Vega E: Th1, Th2, Th17 and Treg levels in umbilical cord blood in preeclampsia. J Matern Fetal Neonatal Med 29: 1642-1645, 2016.
11. Tian T, Yu S, Liu L, Xue F, Yuan C, Wang M, Ji C and Ma D: The profile of $\mathrm{T}$ helper subsets in bone marrow microenvironment is distinct for different stages of acute myeloid leukemia patients and chemotherapy partly ameliorates these variations. PLoS One 10: e0131761, 2015.

12. Kornete M, Mason ES, Girouard J, Lafferty EI, Qureshi S and Piccirillo CA: Th1-Like ICOS ${ }^{+}$Foxp $3^{+} \mathrm{T}_{\text {reg }}$ cells preferentially express CXCR 3 and home to $\beta$-islets during pre-diabetes in BDC2.5 NOD Mice. PLoS One 10: e0126311, 2015.

13. Wang J, Cao H, Wang H, Yin G, Du J, Xia F, Lu J and Xiang M: Multiple mechanisms involved in diabetes protection by lipopolysaccharide in non-obese diabetic mice. Toxicol Appl Pharmacol 285: 149-158, 2015.

14. Takahashi H, Noto YI, Makita N, Kushimura-Okada Y, Ishii R, Tanaka A, Ohara T, Nakane S, Higuchi O, Nakagawa M and Mizuno T: Myasthenic symptoms in anti-low-density lipoprotein receptor-related protein 4 antibody-seropositive amyotrophic lateral sclerosis: Two case reports. BMC Neurol 16: 229, 2016.

15. Rahman MM, Prunte L, Lebender LF, Patel BS, Gelissen I, Hansbro PM, Morris JC, Clark AR, Verrills NM and Ammit AJ: The phosphorylated form of FTY720 activates PP2A, represses inflammation and is devoid of S1P agonism in A549 lung epithelial cells. Sci Rep 6: 37297, 2016.

16. Breart B and Bousso P: $\mathrm{S}_{1} \mathrm{P}_{1}$ downregulation tailors $\mathrm{CD} 8^{+} \mathrm{T}$-cell residence time in lymph nodes to the strength of the antigenic stimulation. Eur J Immunol 46: 2730-2736, 2016.

17. Kaminski HJ, Himuro K, Alshaikh J, Gong B, Cheng G and Kusner LL: Differential RNA expression profile of skeletal muscle induced by experimental autoimmune myasthenia gravis in rats. Front Physiol 7: 524, 2016.

18. Lennon VA, Lindstrom JM and Seybold ME: Experimental autoimmune myasthenia: A model of myasthenia gravis in rats and guinea pigs. J Exp Med 141: 1365-1375, 1975.

19. Meng X, Liu Y, Wang H, Liu L and Gong H: Effcacy of tripterygium glycosides on experimentalautoimmune myasthenia gravis in rats. Int J Clin Exp Med 10: 12235-12239, 2017.

20. Livak KJ and Schmittgen TD: Analysis of relative gene expression data using real-time quantitative PCR and the 2(-Delta Delta C(T)) method. Methods 25: 402-408, 2001.

21. Anand G, Vasanthakumar R, Mohan V, Babu S and Aravindhan V: Increased IL-12 and decreased IL-33 serum levels are associated with increased Th1 and suppressed Th2 cytokine profile in patients with diabetic nephropathy (CURES-134). Int J Clin Exp Pathol 7: 8008-8015, 2014.

22. Dalakas MC: Future perspectives in target-specific immunotherapies of myasthenia gravis. Ther Adv Neurol Disord 8: 316-327, 2015.

23. Luo $\mathrm{J}$ and Lindstrom $\mathrm{J}$ : AChR-specific immunosuppressive therapy of myasthenia gravis. Biochem Pharmacol 97: 609-619, 2015.

24. Yu Y, Cao F, Ran Q and Sun X: Regulatory T cells exhibit neuroprotective effect in a mouse model of traumatic brain injury. Mol Med Rep 14: 5556-5566, 2016.

25. Uzawa A, Kanai T, Kawaguchi N, Oda F, Himuro K and Kuwabara S: Changes in inflammatory cytokine networks in myasthenia gravis. Sci Rep 6: 25886, 2016.

26. Li H, Wang CC, Zhang M, Li XL, Zhang P, Yue LT, Miao S, Wang S, Liu Y, Li YB and Duan RS: Statin-modified dendritic cells regulate humoral immunity in experimental autoimmune myasthenia gravis. Mol Cell Neurosci 68: 284-292, 2015.

27. Fan X, Lin C, Han J, Jiang X, Zhu J and Jin T: Follicular helper $\mathrm{CD} 4^{+} \mathrm{T}$ cells in human neuroautoimmune diseases and their animal models. Mediators Inflamm 2015: 638968, 2015.

28. Yilmaz V, Oflazer P, Aysal F, Durmus H, Poulas K, Yentur SP, Gulsen-Parman Y, Tzartos S, Marx A, Tuzun E, et al: Differential cytokine changes in patients with myasthenia gravis with antibodies against AChR and MuSK. PLoS One 10: e0123546, 2015. 